\title{
A Holographic System for Subsea Recording and Analysis of Plankton and other Marine Particles (HOLOMAR)
}

\author{
Dr John Watson \\ Aberdeen University, \\ Department of Engineering \\ Aberdeen AB24 3UE, Scotland \\ i.watson@eng.abdn.ac.uk
}

\author{
S Alexander ${ }^{\mathrm{a}}$, V Chalvidan ${ }^{\mathrm{b}}$ G Craig ${ }^{\mathrm{a}}$, A Diard ${ }^{\mathrm{c}}$, GL Foresti ${ }^{\mathrm{d}}$, S Gentili ${ }^{\mathrm{d}}$, DC Hendry ${ }^{\mathrm{a}}$, PK Hobson ${ }^{\mathrm{e}}$, RS Lampitt ${ }^{\mathrm{f}}$, H Nareid ${ }^{\mathrm{a}}$, \\ JJ Nebrensky ${ }^{\mathrm{e}}$, A Pescetto ${ }^{\mathrm{g}}$, GG Pieroni ${ }^{\mathrm{d}}$ MA Player ${ }^{\mathrm{a}}, \mathrm{K} \mathrm{Saw}^{\mathrm{f}}$, S Serpico ${ }^{\mathrm{g}} \mathrm{K}$ Tipping ${ }^{\mathrm{f}}$ A Trucco ${ }^{\mathrm{g}}$
} 'Holo 3, 7 rue de General, Saint-Louis 68300 , France, 'Quantel, 17 Ave de l'Atlantique, 91941 Les Ulis, France, ${ }^{\mathrm{d}} \mathrm{Dept}$ of Mathematical \& Information (DIMI),
University of Udine, via del Scienze 206, Udine 33100, Italy, 'Department of Electronic and Computer Engineering, Brunel University, Uxbridge, Middlesex
UB8 3PH, UK, 'Southampton Oceanography Centre, Empress Dock, Southampton SO14 3ZH, UK. 'Dept of Bisphysical \& Electronic Engineering (DIBE),
University of Genova, via all'Opera Pia 11A, Genova I-16145, Italy

\begin{abstract}
We report here details of the design, development, initial testing and field-deployment of the HOLOMAR system for in-situ sub-sea holography and analysis of marine plankton and non-living particles. HOLOMAR comprises a submersible holographic camera ("HoloCam") able to record in-line and off-axis holograms at depths down to $100 \mathrm{~m}$, together with specialised reconstruction hardware ("HoloScan") linked to custom image processing and classification software.

The HoloCam consists of a laser and power supply, holographic recording optics and holographic plate holders, a water-tight housing and a support frame. It utilises two basic holographic geometries, in-line and off-axis such that a wide range of species, sizes and concentrations can be recorded. After holograms have been recorded and processed they are reconstructed in full three-dimensional detail in air in a dedicated replay facility. A computer-controlled microscope, using video cameras to record the image at a given depth, is used to digitise the scene. Specially written software extracts a binarised image of an object in its true focal plane and is classified using a neural network. The HoloCam was deployed on two separate cruises in a Scottish sea loch (Loch Etive) to a depth of $100 \mathrm{~m}$ and over 300 holograms were recorded.
\end{abstract}

\section{Introduction}

The World's oceans and seas are a dominant feature of the planet's ecosystem and contain some of the richest and least exploited reserves of natural resources, but paradoxically are possibly the least understood of our natural habitats. To aid the study of the aquatic environment, it has often been necessary to develop new techniques in measurement and instrumentation. With exploration depths down to $10,000 \mathrm{~m}$ or more, instruments need to be rugged, sensitive, accurate and user-friendly and adaptable to operate on a variety of underwater observation platforms. Over the past 20 years, or so, there has been a dramatic increase in the use of optical techniques for measurement and imaging underwater [1]. As this use of optics has increased so has the quality and reliability of the data received as has our knowledge and understanding of this unique environment. Within this, optical holography [2-29] has shown itself to be especially useful for ron-destructive and non-intrusive interrogation and precision measurement of the proportions and species of plankton within the upper water column.

It can be expected that like conventional optical imaging, holography will see a dramatic increase in its usage as its benefits become more appreciated and the information it yields shows itself to be important. Holography is the only way to record true, full-field, high-resolution, threedimensional optical images. Increasingly, "classical" holographic methods based on photographic recording and "wet" chemical processing are being complemented and replaced by real-time techniques based on all-electronic systems with digital image reconstruction. Electronic (digital) holography also adds the "fourth dimension" through holographic video recording. New. laser technology is opening exciting possibilities in compact low-cost systems.

Conventional methods of sampling plankton are not well suited to observing precise spatial relationships, or preserving some of the more delicate organisms. Holographic recording and imaging offers marine scientists an alternative to conventional imaging for analysis of oceanographic systems. The overriding benefit of holography is that it permits nonintrusive and non-destructive analysis of the organisms and particles in their natural environment, while still preserving their relative spatial distribution. We report here details of the development, initial testing and deployment of the HOLOMAR [3] system for in-situ sub-sea holography and analysis of marine plankton and non-living particles. HOLOMAR comprises a submersible holographic camera ("HoloCam") able to acquire in-line (ILH) and off-axis holograms $(\mathrm{OAH})$ at depths down to $100 \mathrm{~m}$, and specialised reconstruction hardware ("HoloScan") linked to custom image processing and classification software. 


\section{History of Holography of Marine Organisms}

The first known use of holography for recording living marine plankton was by Knox in 1966 [4], who recorded inline holograms (ILH), using a short coherence length ruby laser, of a variety of living marine plankton species in a tank. However to avoid problems with refractive index and wavelength changes he immersed the holographic plate directly in the water, which is completely impractical for field use. Later, Beers et al, [5] carried out similar work using a pulsed argon-ion laser $(514 \mathrm{~nm})$. The first papers to report quantitative data from replayed holograms of marine particles recorded in situ were those of Carder and co-workers $[6,7,8]$. They used in-line holography to record mainly abiotic oceanic sedimenting material. Small volumes $\left(10\right.$ or $\left.20 \mathrm{~cm}^{3}\right)$ were recorded using the in-line technique and a low power HeNe laser.. Multiple time-lapse holograms were recorded at $0.3 \mathrm{~s}$ or $5 \mathrm{~s}$ intervals. The use of a low power continuous wave (c.w.) laser limited the technique to slowly moving objects.

Heflinger and co-workers $[9,10]$ used the off-axis geometry with both pulsed argon-ion $(514 \mathrm{~nm})$ and pulsed Xenon $(535 \mathrm{~nm})$ laser to record plankton on $35 \mathrm{~mm}$ film. In order to completely compensate for the aberrations introduced by replaying the real image in air, they replayed the real image back into the water tank using a true conjugate beam from a continuous-wave argon laser. The sides of their tank were flexible so that the real image could be placed close to the exit window and thus viewed via an external microscope at high resolution. This technique of replaying the real image into a water tank does pose considerable problems for automated analysis of large volumes of water and is not considered to be really practical, however the resolution obtained was extremely impressive, especially for the off-axis technique.

The first development of a subsea holographic camera appears to have been that by Stewart, Beers and Knox [11] who deployed an in-line holocamera from the $R V$ Ellen $B$ Scripps. The laser was a pulsed Xenon laser and had variable optical paths lengths in water of 1,2 , or $3 \mathrm{~m}$, with water volumes up to $10^{5} \mathrm{~cm}^{3}$. The camera was designed to operate to a depth of $100 \mathrm{~m}$ in water, with holograms being recorded as the instrument was lowered at a rate of around $0.03 \mathrm{~m} \mathrm{~s}^{-1}$. No data is presented in their paper.

O'Hern, d'Agostino and Acosta [12] recorded larger volumes of water using a ruby laser. Their in-line camera was used to measure the sizes of cavitation nuclei in sea-water to a maximum depth of $33.5 \mathrm{~m}$. Hologram replay was with a $\mathrm{HeNe}$ laser and a resolution of $5 \mu \mathrm{m}$ quoted. Tens of holograms were recorded at two sites near Santa Catalina Island off Los Angeles, USA. Costello et al [13] used a holographic sedimenting trap which demonstrated a resolution of $12 \mu \mathrm{m}$ in a few cubic centimetres volume. Their system allowed two in-line holograms to be recorded simultaneously from two orthogonal directions. Four sedimenting traps were deployed on three cruises in the North Pacific Ocean and over 4000 holograms were recorded. Their replay system used a combination of direct projection on to a flat screen that was viewed directly by an observer plus a video camera whose output was digitised for image processing. The holograms were replayed with a $\mathrm{HeNe}$ laser $(632.8 \mathrm{~nm})$ rather than the more ideal Ar-ion at $514 \mathrm{~nm}$. This suggests that either they were unaware of the opportunity to compensate for aberrations by changing the replay wavelength, or that other factors (not mentioned in the paper) led to the use of the HeNe laser. This work is notable for being deployed in the field and for collecting a large sample of data, but the authors make the telling comment that the large volume of data generated present, notables difficulties requiring further attention.

The first really successful underwater holographic cameras for plankton recording, were developed by Katz and his team, at Johns Hopkins University, Baltimore, USA [1416] whose work sprung from an interest in fluid dynamics. The latest version is based on a ruby laser and is designed to record in-line holograms on rolls of film in a $63 \mathrm{~mm}$ diameter sample volume that can be varied from 100 to $680 \mathrm{~mm}$ in length. The camera utilised a multi-tube design with one tube holding the laser and optics and another containing power units. The recording volume is situated $890 \mathrm{~mm}$ above the camera to minimise disturbance to flow. The camera is designed as a "Lagrangian drifter" and can drift with the water current. The camera also utilised film rather than plates because of the reduced resolution requirements of in-line holography. The camera was successfully deployed in, for example, Chesapeake Bay, Baltimore and Solomons Island, Maryland. Holograms were recorded of various species of phytoplankton and zooplankton and represented a significant advance in the deployment of sub-sea holography. Field trials demonstrated the reliability and resolution of the system. Particles as small as $10 \mu \mathrm{m}$ were identified [15]. Currently analysis of the digitised images is performed manually, and this is described as "labor intensive and time consuming". Not surprisingly a key goal for future work is to automate this task [16].

From the early 1980's, teams lead by Watson in Aberdeen, Scotland investigated the use of underwater holography for applications in the offshore oil and gas industry $[2,17,18]$. Primary interest was in inspection of pipelines for corrosion and damage and much of the work concentrated on an analysis of the optical aberrations introduced by recording in water and replaying in air and methods of compensating for such aberrations [19-21]. Later, in collaboration with workers from Southampton Oceanography Centre and Brunel University in England, this work was adapted for holography of plankton [22-24] and the consequent development of the HoloMar system [3,23-27]. Unlike Katz, Watson used a (green) frequency-doubled NdYAG laser (for optimal transmission through water) and is the first system to incorporate simultaneous ILH and OAH recording. Both Katz [15,16] and Watson holocameras [27] have been deployed in a variety of subsea conditions to record high-resolution images of plankton and other marine particles to depths of $100 \mathrm{~m}$ and more from which $3 \mathrm{D}$ distribution data of plankton has been obtained. It is this sytem that we describe in the remainder of this paper. 
More recent developments have utilised, digital holographic recording in the in-line mode [28,29], and it is to be expected that much more use will be made of "eholography" in the future.

\section{Methodology of Holographic Recording and Replay of Plankton}

In the HoloMar system, holograms of aquatic systems are recorded in-situ using a specially developed subsea holographic camera (HoloCam). Holograms can be recorded, simultaneously, in either the in-line reference beam (ILH) or off-axis reference beam (OAH) geometry (see below). Although the recording of the holograms take place in water, replay of the image is carried out in air, in the laboratory, to provide identification and measurement of the organisms and particles. The two modes of operation have distinct differences and advantages.

In-line reference beam holographic recording (ILH): A single laser beam is directed through the sample volume towards the holographic plate and records the optical interference between light diffracted by the object and the undeviated portion of the illuminating beam. The replayed hologram simultaneously forms two images located on the optic-axis which for a collimated beam are at equal distances on either side of the holographic plate. The organisms are illuminated in transmission and the scene should have an overall transparency of about $80 \%$ so that speckle noise does not seriously degrade the image quality. This criterion sets an upper limit on the recordable particle concentration at around 40 particles $\mathrm{cm}^{-3}$ (for $20 \mu \mathrm{m}$ diameter particles and a recorded depth of $1 \mathrm{~m}$ ). There is also a need to balance the size of the particles with the object-to-hologram distance. The upper limit for good imaging is a few millimetres; however, particles down to around $5 \mu \mathrm{m}$ dimensions can be identified and measured with ease.

Off-axis reference beam holographic recording (OAH): A two-beam geometry is utilised. One beam illuminates the scene and the other directly illuminates the holographic film at a known incidence angle. Interference occurs between diffuse light reflected from the scene and the angularly separate reference beam. On replay, the real and virtual images are similarly spatially separated which makes their interrogation easier. Off-axis holography is usually applied to, primarily, opaque subjects of large volume, making it better suited to recording of more dense aggregates of marine particles. The scene can be front, side or back illuminated (or some combination of all three). Although there is no real upper limit to the size of particles that can be recorded (this is determined by available energy and coherence of the laser) the practical lower limit to the resolution that can be achieved is less than $100 \mu \mathrm{m}$.

Replay of holograms and data extraction: After wet chemical processing of the holographic plate to preserve the interference pattern, the holograms are replayed in air, in the laboratory, using the real (projected) image mode of reconstruction. The illuminating beam is normally the phase conjugate of the original reference beam used in recording. Holograms are replayed in either in-line or off-axis modes according to recording. A video camera (with or without lens, as appropriate) is mounted on computer-controlled $x-y-z$ stepper stages. The camera is translated through the image to extract information regarding shape, identity, dimensions and relative position. Image analysis and data extraction facilities allow species identification and measurement of local concentration of a variety of marine organisms. High resolution, 3-dimensional images of the recorded underwater scene are recreated in the laboratory and located in the realimage space in front of the observer. Images are directly interrogated by measuring microscopy or video to extract information at any point in an individual plane of the image to give the dimensions, shape, identity and relative position of the particles. This ability to "optically section" the image is what sets holography apart from standard photography or stereo photogrammetry. Since a pulsed laser with short pulse duration is used for the recording, the object scene is effectively frozen at the recording instant allowing even fast moving particles to be recorded. The spatial distribution and relative location of the particles can be analysed as well as the individual particles. Detail of less than $10 \mu \mathrm{m}$ can be resolved in volumes up to $50,000 \mathrm{~cm}^{3}$ (depending on mode of operation). Since the holograms are recorded on photographic emulsion a permanent archive is obtained.

A key issue in the analysis and replay of holograms recorded in water is the severe nature of the optical aberrations introduced when they are replayed in air. Other workers have employed methods such as immersing the holographic plate in water during recording [4] or direct replay back into water [9]. Our approach to compensating for the refractive index misrnatch is to replay the holograms at the effective wavelength of the laser in water, rather than at the recording wavelength. However, to fully optimise this compensation method reciuires that the optical path lengths of the light in water, through the observation window and inside the camera also have to be taken into account. This determines that for our camera configuration (recording at $532 \mathrm{~nm}$ ), the holograms need to be replayed at a wavelength between $440 \mathrm{~nm}$ and $520 \mathrm{~nm}[19,20,21]$.

\section{System Configuration and Operation}

The complete HOLOMAR system consists of two main parts: the HoloCam for recording of the holograms and the reconstruction facility, HoloScan, for replay, data extraction and analysis.

\section{A. The Recording System (HoloCam)}

Fig. 1 (end of paper) shows the HoloCam layout and configuration. The laser is a Q-switched, frequency-doubled Nd-YAG with an output energy of $700 \mathrm{~mJ}$ in a single pulse of less than $10 \mathrm{~ns}$ duration. The wavelength is $532 \mathrm{~nm}$ (chosen to coincide with the optimum transmission window of seawater) and operates in a single longitudinal mode with a coherence length in excess of $2 \mathrm{~m}$. The laser and optical baseplates are manufactured from the same aluminium alloy to eliminate problems due to thermal expansion. The power supplies and cooling system are located beneath the main optical baseplate on a secondary base. 
The laser produces two output beams: one of $100 \mathrm{~mJ}$ and the other of $600 \mathrm{~mJ}$ energy. The output from the laser is linearly polarised, in a horizontal plane but transport through the beam steering mirrors effectively rotates this, so that holographic recording occurs with vertically polarised light. The $100 \mathrm{~mJ}$ beam is split into two roughly $50: 50$ beams at the beam splitter (BS). The straight through path forms the illuminating beam for the in-line mode and the reflected path forms the reference beam for the off-axis mode. Both beams are expanded and collimated using Galilean-type beam expansion optics to a diameter of $100 \mathrm{~mm}(\lambda / 8$ wavefront flatness over the whole aperture). The in-line beam passes into the starboard arm before being directed through a window into the water, through another window and onto the hologram plate located in the opposite arm. All windows are high quality optical flats $(\lambda / 4)$. The off-axis beam passes through beam steering and path-length compensation assemblies, before collimation to form the reference beam. The off-axis beam is then folded at a mirror and is incident on the plate at $60^{\circ}$ to the normal.

The arrangement of in-line and off-axis holographic geometries allows simultaneous recording of partially overlapping volumes of water, to provide an element of cross-correlation between holograms. The in-line path records a water column of $470 \mathrm{~mm}$ by $90 \mathrm{~mm}$ diameter at $400 \mathrm{~mm}$ from the front face of the housing, whereas the offaxis hologram records almost the entire volume $\left(50,000 \mathrm{~cm}^{3}\right)$ delineated by the front window to the end of the arms. For illumination of the off-axis volume, side illumination provides the best images when recording plankton. A novel "lightrod" has been devised to provide roughly even side illumination to the off-axis volume. This encompasses a hollow perspex tube with 10 glass plates distributed equally along the length, positioned at $45^{\circ}$ to the main axis. Each surface acts as a partial reflector, deflecting about $9 \%$ of the beam sideways into the recording volume, the last plate is a total reflector. Three lightrods are mounted on the outside of the camera (two starboard, one port), with the $600 \mathrm{~mJ}$ laser output being split evenly between them.

Two motor-driven plate holders and transport mechanisms were developed. For space reasons the in-line holder holds 20 plates and the off-axis holds 25 . The glass holographic plates are located in plastic frames and stacked in a detachable cassette. The plastic frames protect the glass plates and provide a mechanical interface for the plate extraction and movement mechanisms. The cassettes ensure no exposure to stray light and allow the plates to be easily transported to and from the darkroom processing facility. The control sequencing is through software, running on the micro-controller system described below. One plate can be exposed every 10 seconds.

The topside control console (PC) runs the main control program (Windows based written in Visual $\mathrm{C}++$ ) providing a user interface as well as displaying system information and storing data for each recording. A network of 4 microcontroller boards (Siemens C167CR) serves as the backbone of the in-camera control system. Communication between topside and the camera modules is via a main umbilical cable using the CAN bus protocol. All micro-controller code is written in $C$, and designed so that micro-controller actions and responses are driven by simple CAN messages. The first and second micro-controllers control, respectively, the in-line and off-axis plateholder mechanisms. The third microcontroller is responsible for internal and external sensors, comprising internal temperature, humidity and tilt sensors interfaced directly to the micro-controller, and an external CTD system communicating via an RS232 link. The fourth micro-controller drives the laser via a second RS232 link.

The entire camera is enclosed in a water-tight stainless steel housing and the overall dimensions are $1000 \mathrm{~mm}$ diameter by $2200 \mathrm{~mm}$. The initial prototype system is capable of either ship deployment or attachment to a fixed buoy and allows recording down to a depth of $100 \mathrm{~m}$. A CTD (conductivity-temperature-depth) profiler can be mounted on the frame together with a conventional video system to allow observation of the scene prior to hologram recording.

Figs 2 and 3 show views of the inside and the front end of HoloCam.

\section{B. The Replay and Data Extraction System (HoloScan)}

Precision replay of the holograms is accomplished in a dedicated reconstruction facility comprising laser, reconstruction optics and image acquisition and analysis instrumentation and software.

Fig. 4 shows a schematic of the replay system for in-line and off-axis modes. The replay laser (Kimmon $180 \mathrm{~mW}$, $442 \mathrm{~nm} \mathrm{HeCd}$ ) is mounted along one arm above the collimating optics. The collimating optics consists of a Keplerian telescope (CVI $1000 \mathrm{~mm}$ focal length achromatic objective and a Melles-Griot $10 \mathrm{~mm}$ doublet) producing a $100 \mathrm{~mm}$ diameter beam flat to $\lambda / 5$. The plateholder is located near the vertex end of the stage arm and by moving a large front-surface rectangular mirror (flat to $\lambda / 10$ ) between two fixed positions along the collimated beam it is possible to rapidly change the replay angle between the in-line (normal to plate) and off-axis configurations. The hologram can also be precisely rotated about the $y$-axis. A set of computercontrolled stepper-stages (Ealing DPS system, $1000 \mathrm{~mm}$ stage in $10 \mu \mathrm{m}$ steps and two $200 \mathrm{~mm}$ stages in $5 \mu \mathrm{m}$ steps) carrying a videocamera (JAI CV-M300 CCD) is mounted along the other arm. Although the objects are located in water, image replay is carried out in the laboratory in air using the projected (real) image mode of reconstruction. This change in refractive index can introduce significant aberrations, particularly with off-axis holography, which may be corrected for by replay at a shorter wavelength. The HoloCam's window thickness and air gap to the plate have been optimised for reconstruction with $442 \mathrm{~nm}$ illumination from a $\mathrm{HeCd}$ laser. 


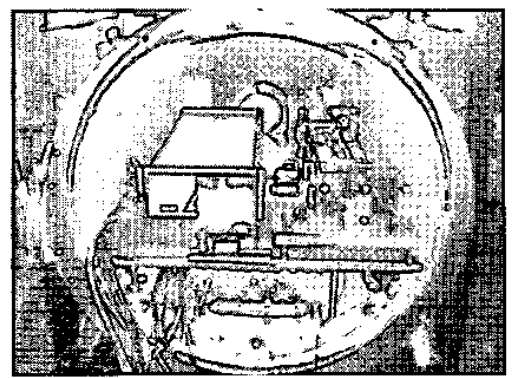

Fig. 2: HoloCam rear (inside)

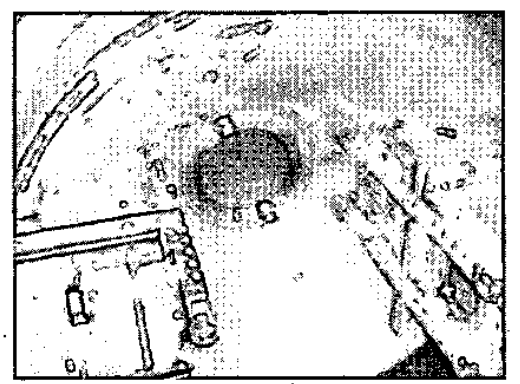

Fig. 3: HoloCam front end

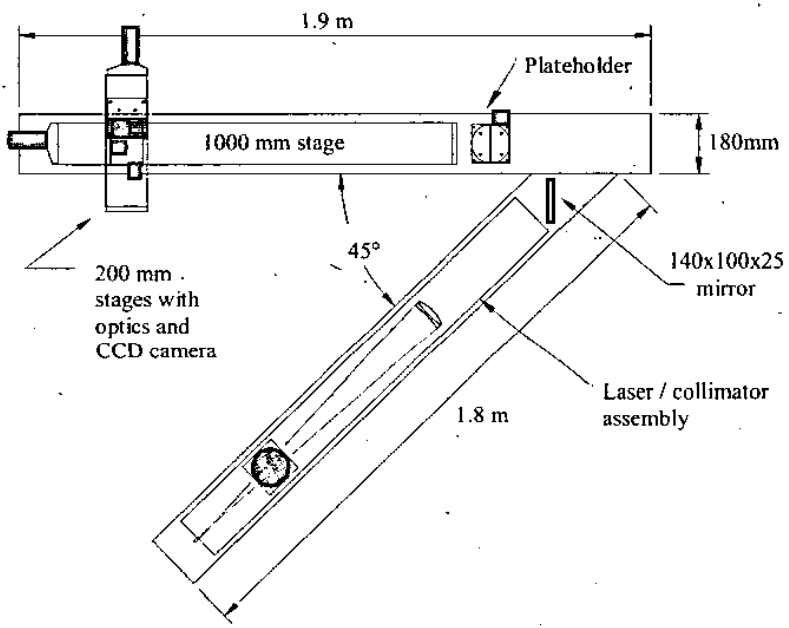

Fig. 4: 'HoloScan' hologram replay machine (off-axis configuration)

The extraction of species distribution data is separated into three steps. Firstly, the videocamera is scanned through the depth of the sample volume in a series of $0.1 \mathrm{~mm}$ steps. When the end is reached the camera is panned sideways before returning to eventually scan the entire volume. For each step, the corresponding video output is captured by an ITI PC-Vision frame-grabber and then fed to the integrated processing software for image enhancement and object focusing within the 3-dimensional sample volume.

The integrated suite of software algorithms perform lowlevel (noise cleaning and image enhancement), middle-level (image segmentation, binarisation, object localisation and tracking) and high-level (3-d information extraction) processing procedures on the holographic image sequence.

Figs 5 and 6 (at end of paper) show a set of processing sequences which illustrate these procedures. The binary images of each organisrn that are generated by this operation are fed to the classification software which is based on the "Hu moments" system of characterisation. Five different neural networks, characterised by different structures, training sets and features, were trained by a "Back Propagation" algorithm for phytoplankton classification from in-line images. The network was trained by patterns containing the first five Hu moments, a number depending on the organism elongation along the major inertia axis and a number depending on the ratio of the organism elongation along the secondary and the major inertia axes. The network structure consists of an input layer of 7 neurons, a hidden layer of 7 neurons and an output layer of 5 neurons corresponding to the four classes of phytoplankton (Asterionella, Ceratium, Diatom and Floc) and to the zooplankton class. For off-axis holograms the recognition is limited to copepods and crustaceans.

\section{System evaluation}

The HoloCam unclerwent a series of laboratory (at Aberdeen) and tank and dockside trials (at Southampton Oceanography Centre (SOC)) prior to undergoing sea trials on board the $R V$ Calanus (Dunstaffnage Marine Lab, Oban, Scotland). The camera was launched in Loch Etive (Fig. 4), a sea loch on the west coast of Scotland, on three separate dives over October/November 2000. A total of 54 pairs of holograms (one in-line and one off-axis) were successfully recorded between the surface and $100 \mathrm{~m}$ depth at 5 or $10 \mathrm{~m}$ interval. Another cruise one year later (September/October 2001), at the same location produced a further one hundred holograms. Qualitative examination of the holograms showed that they were of a high and consistent recording quality. All the holograms were recorded on Agfa Millimask plates. Although these high-resolution (about $4000 \mathrm{~mm}^{-1}$ resolving power) silver halide emulsions were developed for lithographic applications they are particularly suitable for holographic recording; their peak sensitivity is in the green thereby matching our recording wavelength of $532 \mathrm{~nm}$. Wet chemical development was in undiluted Tetenal Neofin Blue for $2 \mathrm{~min}$ at $20^{\circ} \mathrm{C}$ followed by fixing for $2 \mathrm{~min}$.

Visual inspection of the unmagnified virtual image (offaxis) revealed each hologram to contain varying numbers of marine organisms and particles. Real image evaluation of both in-line and off-axis holograms confirmed the initial findings. Qualitatively, the highest concentrations of marine organisms were at the greater depths (below about $40 \mathrm{~m}$ ). The holograms of one dive, taken as night was falling, appeared to show that the plankton were moving higher in the water column. 


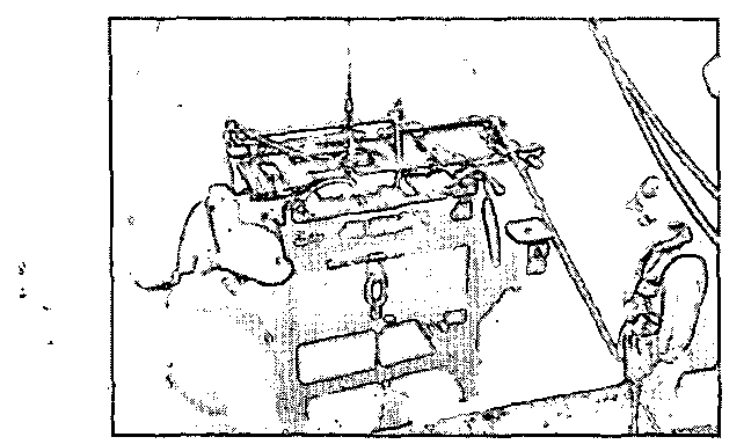

Fig 7: HoloCam being lowered into Loch Etive.

Figs 8 and 9 (below) show sample images taken from inline and off-axis holograms, respectively. They have been identified to be calanoid copepods in either Stage $\mathrm{V}$ of development or adults. The in-line holograms reveal the presence of large numbers of organisms in the size range from $0.5 \mathrm{~mm}$ to a few millimetres. In each of these holograms there were significant amounts of detritus and other particles below $100 \mu \mathrm{m}$ dimension. Detail in these images could be seen to better than $10 \mu \mathrm{m}$ resolution. The same observations are apparent in the off-axis holograms, but the images show lower resolution and are more sensitive to orientation of the organism (the entire organism cannot be in focus at once). The in-line holograms show essentially outlines or silhouettes of the objects, while the off-axis holograms show the whole illuminated surface of the organism.

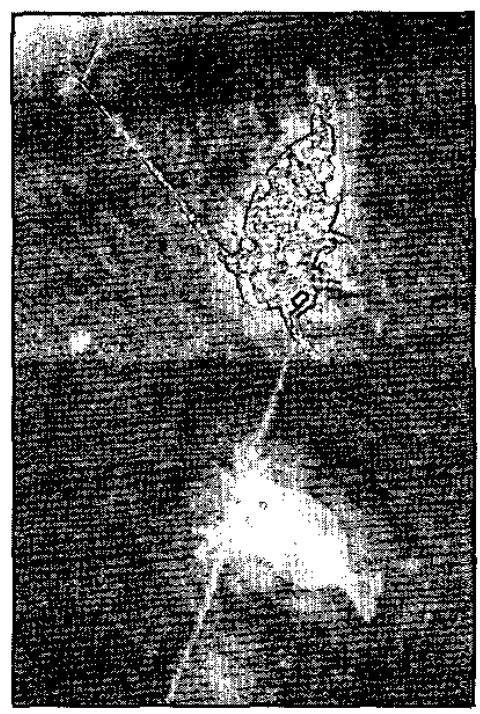

Fig 8: Images of calanoid copepods from in-line holograms recorded at $70 \mathrm{~m}$ and $80 \mathrm{~m}$ depth respectively. Each organism is approximately $2 \mathrm{~mm}$ long.

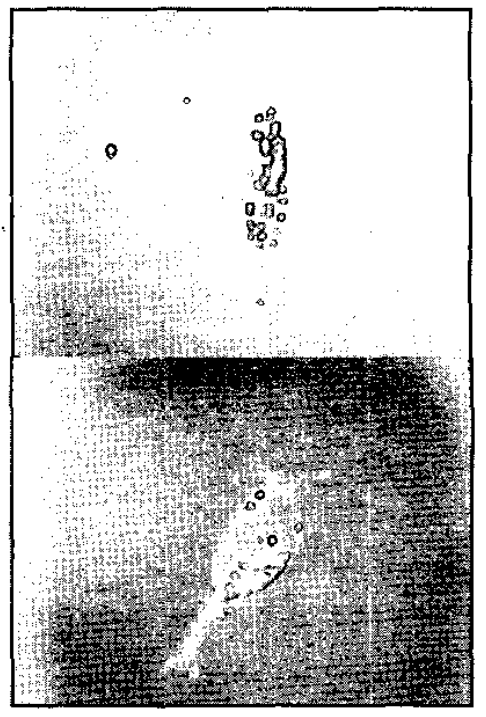

Fig 9: Images of calanoid copepods taken from off-axis holograms. Each organism is about $6 \mathrm{~mm}$ long and were recorded at $60 \mathrm{~m}$ depth (in the same hologram)

Detailed quantitative analysis of the holograms to identify the organisms and extract concentrations and relative distribution requires that the holograms be precisely replayed in HoloScan and the processing software applied. There is now an excellent collection of "live" holograms from which information can be extracted. It can be stated unequivocally that the holograms from SOC and Loch Etive contain outstanding images of plankton and marine snow particles. The clarity of the images is very high and the holograms are being analysed for species, concentration and 3-dimensional coordinates using HoloScan. The data from this study will be published in due course.

\section{Conclusions}

HOLOMAR can, unconditionally, be described as a scientific and technical success. A unique camera (HoloCam) has been designed and built which can record simultaneous in-line and off-axis holograms. The camera has been deployed from a ship and operated to a depth of $100 \mathrm{~m}$. A set of several hundred holograms at 5 or $10 \mathrm{~m}$ depth intervals has been recorded. On qualitative examination, these holograms are seen to be rich in plankton and other marine particles. The associated replay facility (HoloScan) is itself a state-of-theart system, which allows the extraction of images from the holograms. The images can be optimised to produce as high a resolution as possible; they are then captured and stored prior to image enhancement and classification by the processing software (HoloProc). A series of further sea trials are anticipated and the data will be banked with the European Commission. 


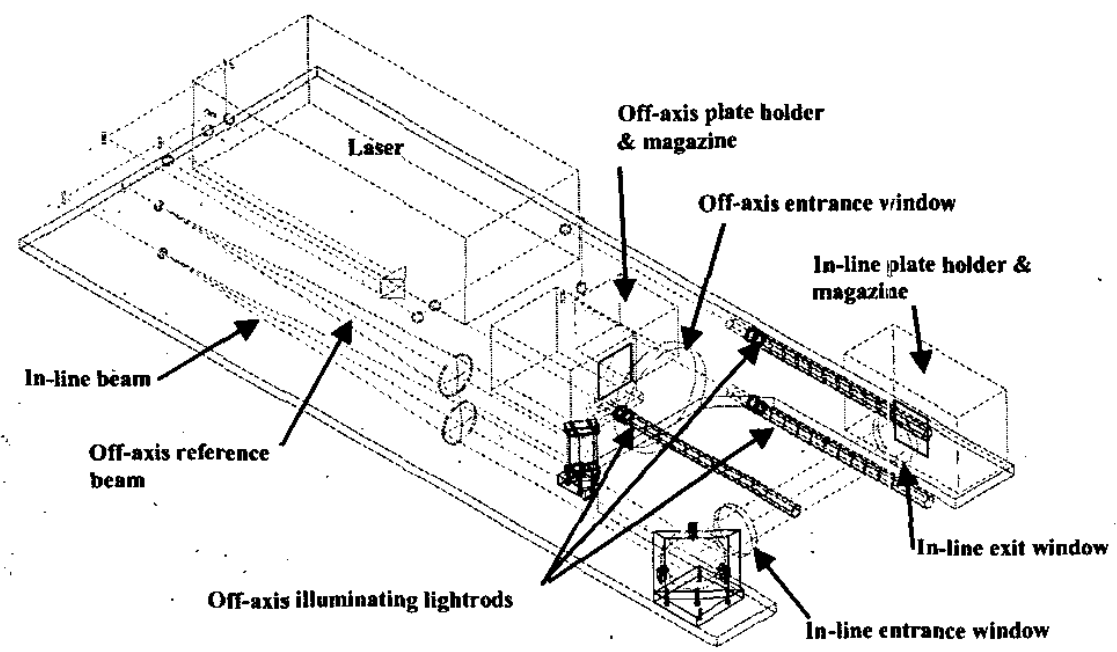

Fig. 1: The Layout of HoloCam showing both in-line and off-axis paths

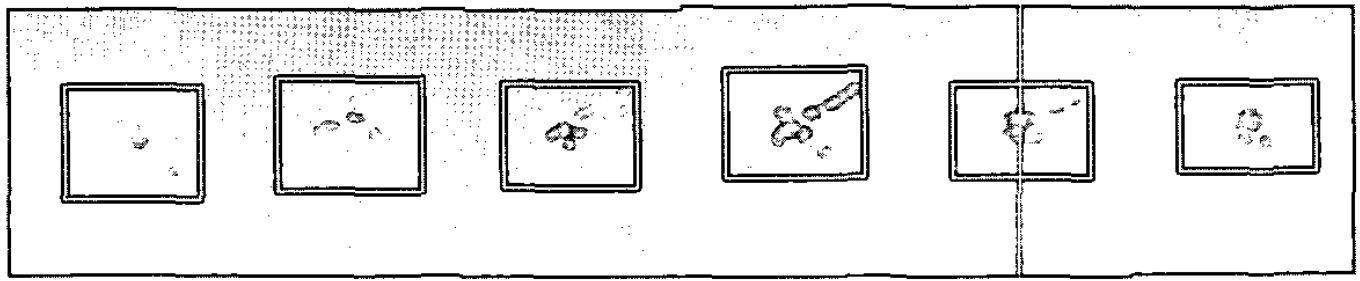

Fig 5: A sequence of images of Asterionella formosa shown in depth order through the position of best focus

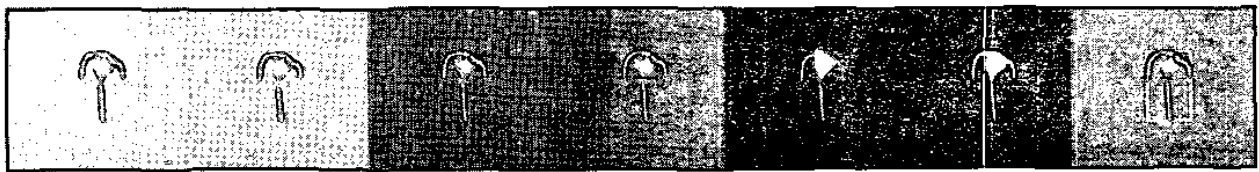

Fig 6: A sequence of images of ceratium showing, from left to right, (a) image at best focus, (b) after stop band filtering, (c) histogram manipulation, (d) median filtering, (e) segmentation, (f) binarisation $(\mathrm{g})$ noise filtering and localisation

\section{Acknowledgements}

The authors wish to acknowledge the support of the European Commission in bringing this work to fruition (EC MAST-III Project No. MAS3-CT97-0079). They also wish to thank their respective institutions for their encouragement in this work.

\section{References}

[1] M Solan et al, "Towards a greater understanding ...... a picture is worth a thousand worms" J Exp Mar.Biol
Ecol, 285-286, 313-338 (2003)

[2] J. Watson, "Hologrammetry and its applications", $J$. Imaging Tech. 15, 38-46, (1989)

[3] HoloMar - MAS3-CT97-0079 (EC MAST-III 1997)

[4] C. Knox, "Holographic microscopy as a technique for recording dynamic microscopic subjects", Science 153, 989-90, (1966)

[5] Beers J R, Knox C and Strickland J D H "A permanent record of plankton samples using holography" Limnol. Oceanogr. 15: 967-970, (1970) 
[6] Carder, K.L. Holographic microvelocimeter for use in studying ocean particle dynamics, Opt.Eng. 18 524-525, (1979)

[7] Carder KL, Meyers DJ, "Holography of settling particle shape parameters" Opt. Engng 19, 734-738, (1980)

[8] K.L. Carder, R.G. Steward \& P.R. Betzer, "In situ holographic measurements of the sizes and settling rates of oceanic particulates", J. Geophys. Res.-Oc. Atm. 87, $5681-5685,(1982)$

[9] L.O. Heflinger, G.L. Stewart \& C.R. Booth, "Holographic motion pictures of microscopic plankton", Appl. Opt. 17, 951-954, (1978)

[10] Briones RA, Heflinger LO, Wuerker RF, "Holographic microscopy" Applied Optics 17: 944-950, (1978)

[11] Stewart GL, Beers JR and Knox C; "Application of holographic techniques to the study of marine plankton in the field and the laboratory" Proc SPIE 41, 183-188, (1970)

[12] P O'Hern TJ, L d'Agostino, Acosta AJ "Comparison of Holographic and Coulter Counter measurements of cavitation nuclei in the ocean" Trans ASME J.Fluids Eng 110: 200-207, (1988)

[13] DK Costello et al, "In situ holographic imaging of settling particles: applications for individual particle dynamics and oceanic flux measurements", Deep-Sea Research 36, 1595-1605, (1989)

[14] J. Katz, T.J. O'Hern \& A.J. Acosta, “An underwater holographic camera system for detection of microparticles", Proc. ASME Cavitation and Multiphase Flow Forum, New Orleans, (1984)

[15] J Katz et al "Submersible holocamera for detection of particle characteristics and motions in the ocean", DeepSea Res. 46, 1455-1481, (1999)

[16] E Malkiel, O Alquaddoomi and J Katz, et al, “ Measurements of plankton distribution in the ocean submersible holography", Meas. Sci. Technol 10, 1141 1152 (1999)

[17] Watson J "Underwater visual inspection and measurement using optical holography", Optics and Lasers in Engineering, 16, 375-390, (1992)

[18] P.R. Hobson \& J. Watson, "Accurate three-dimensional metrology of underwater objects using replayed real images from in-line and off-axis holograms" Meas. Sci. Technol. 10, 1153-1161, (1999)

[19] J M Kilpatrick and J Watson, "Underwater Hologrammetry: Reduction of Aberrations by Index Compensation" J.Phys.D: Appl.Phys, 26, 177-182, (1993)

[20] J M Kilpatrick and J Watson "Precision replay of underwater holograms" Meas Sci Technol 5, 716-725, (1994)

[21] J Watson and..J M Kilpatrick "Optical aberrations in underwater holography and their compensation", Proc. SPIE Practical Holography V, Vol. 1461, 245-253, (1991)

[22] J Watson et al, "Holographic mensuration of suspended particles in aquatic systems", Proc.SPIE 2577: 191-199, (1995)

[23] J Watson et al, "Simultaneous in-line and off-axis subsea holographic recording of plankton and other marine particles", Meas Sci Technol 12, L9-L15, (2001)

[24] PR Hobson and J Watson, "The principles and practice of holographic recording of plankton", Jnl of Optics A: Pure and Applied Optics, 4, S34-S49 (2002)

[25] J Watson et al "High resolution in situ holographic recording and analysis of marine organisms and particles (HOLOMAR)" Proceedings of Oceans '98, 1599-1603, (1998)

[26] J Watson et al, "HoloCam: A subsea holographic camera for recording marine organisms and particles" Optical Diagnostics in Engineering, NA Halliwell (Ed), Proc SPIE Vol 4076, 111-119 (2000)

[27] J Watson et al, "Simultaneous in-line and off-axis subsea holographic recording of plankton and other marine particles", Meas Sci Technol (Formerly J Phys E) 12, L9-L15, (2001)

[28] R B Owen and A A Zozulya "In-line digital holographic sensor for monitoring and characterizing marine particulates", Opt. Eng. 39 2187-97 (2000)

[29] H Sun, et al, "In-line digital holography for the study of erosion processes in sediments", Meas. Sci \& Tech 13, L1-L6, (2002) 